\title{
MIS-Tree Algorithm for Mining Association Rules with Multiple Minimum Supports
}

\author{
M. Sinthuja, S. Sheeba Rachel and G. Janani
}

\begin{abstract}
Mining association rules with multiple minimum supports are an important generalization of the association-rule-mining problem. Instead of setting a single minimum support threshold for all items, they allow users to specify multiple minimum supports to reflect the natures of the items, and an Apriori-based algorithm, named MSapriori, is widely used to mine all frequent itemsets. In this paper the FP-tree-like structures, MIS-tree is widely used to store the crucial information about frequent patterns. The benefit of MIS-tree is it avoids rescanning and finds the different frequent itemsets.
\end{abstract}

Keywords--- Data mining, Association rules, Minimum Support, FP-Tree

\section{INTRODUCTION}

$\mathrm{D}$ ATA MINING is the discovery of hidden information found in database and can be viewed as a step in knowledge discovery process. It is motivated by decision support problem faced by most retail organizations. The main challenges in database mining are developing fast and efficient algorithms that can handle large volumes of data. One of the most important data mining applications is that of mining association rules. Data mining has recently attracted considerable attention from database practitioners and researchers because of its applicability in many areas such as decision support, market strategy and financial forecasts. Many approaches have been proposed to find out useful and invaluable information from huge databases. One of the most important approaches is mining association rules [1].

Association rule mining is one of the important techniques of data mining. It aims to extract intresting correlations, frequent patterns, associations or casual structures among a large set of data items. Association rules that can be stated as follows. Let $I=\{i 1, i 2, \ldots, i \mathrm{~m}\}$ be a set of items and $D$ be a set of transactions, where each transaction $\mathrm{T}$ (a data case) is a set of items.

An association rule is an implication of the form, $\mathrm{X} \rightarrow \mathrm{Y}$, where $X \rightarrow I, Y \rightarrow I$ and $X^{\wedge} y=\Phi$. The two basic parameters of Association Rule Mining (ARM) are
a) Support
b) Confidence

M. Sinthuja, E-mail: sinthujamuthu@gamil.com S. Sheeba Rachel, E-mail: s.sheebarachel@gmail.com

G. Janani, E-mail: jananisrit@gmail.com
Support(s) of an association rule is defined as the percentage/ fraction of records that contain XUY to the total number of records in the database. Support $(s)$ is calculated by the following formula:

$$
\text { Support }(X Y)=\frac{(\text { Support count of } X Y)}{(\text { Total number of transaction in D) }}
$$

Confidence of an association rule is defined as the

Percentage/ fraction of the number of transactions that contain XUY to the total number of records that contain X, where if the percentage exceeds the threshold of confidence an interesting association rule $\mathrm{X}=>\mathrm{Y}$ can be generated.

$$
\text { Confidence }(X, Y)=(\text { Support }(X Y))
$$

$$
(\operatorname{Support}(X))
$$

The key element that makes association-rule mining practical is minsup. It is used to prune the search space and to limit the number of rules generated. However, using only a single minsup implicitly assumes that all items in the database are of the same nature or of similar frequencies in the database. This is often not the case in real-life applications [2] [12].

Association rule model allowed the user to specify multiple minimum supports to reflect different natures and frequencies of items [12]. Specifically, the user can specify a different minimum item support for each item. Thus, different rules may need to satisfy different minimum supports depending on what items are in the rules. This model enables users to produce rare item rules without causing frequent items to generate too many meaningless rules. However, the proposed algorithm named the MSapriori algorithm [12], adopts an Apriori-like candidate set generation- and-test approach and it is always costly and time-consuming. The next proposed algorithm multiple item support tree (MIS-tree for short) structure, which extends the FP-tree [4] structure for storing compressed and crucial information about frequent patterns.

\section{LITERATURE SURVEY}

Throughout this paper consider the transaction table 1 . This section mainly focused on the mining association rules with multiple minimum supports. Basic algorithms of association rule mining is Apriori algorithm and FP-growth 
tree.

\subsection{Apriori Algorithm}

The Apriori algorithm discovers frequent itemsets from databases by iteration. Basically, iteration i computes the set of frequent i-itemsets (frequent patterns with $\mathrm{i}$ items). In the first iteration, the set of candidate 1-itemsets contains all items in the database [2]. Then, the algorithm counts their supports by scanning the database, and those 1-itemsets whose supports satisfy the MS threshold are selected as frequent 1 -itemsets. In the kth (kz2) iteration, the algorithm consists of two steps. First, the set of frequent itemsets Lk_1 found in the (k_1) th iteration is used to generate the set of candidate itemsets $\mathrm{Ck}$. Next compute the supports of candidate itemsets in $\mathrm{Ck}$ by scanning the database and then we obtain the set Lk of frequent k-itemsets. The iteration will be repeatedly executed until no candidate patterns can be found.

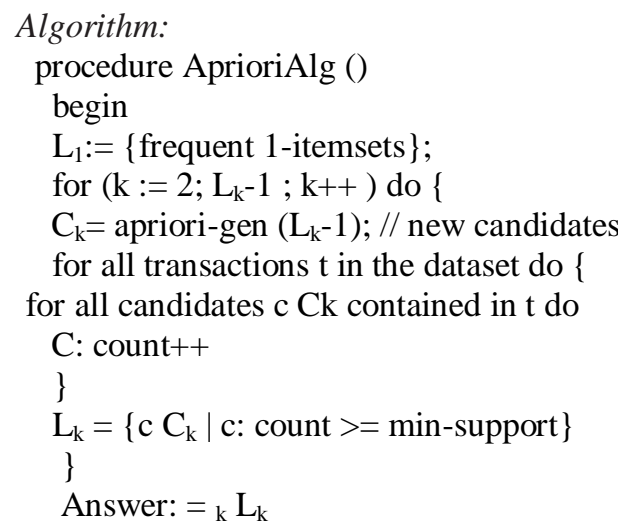

End

Similarly, frequent k-itemset can be found until there is no frequent itemsets.

Figure 1 shows the steps involved to find the frequent itemsets.

Table 1: Transaction Database

\begin{tabular}{|l|c|}
\hline TID & Items \\
\hline 100 & a, c, d, f \\
\hline 200 & a, c , e, f, g \\
\hline 300 & a, b, c, f, h \\
\hline 400 & b, f, g \\
\hline 500 & b, c \\
\hline
\end{tabular}

\begin{tabular}{|l|l|}
\hline Itemset & count \\
\hline$\{a\}$ & 3 \\
\hline$\{b\}$ & 3 \\
\hline$\{c\}$ & 4 \\
\hline$\{d\}$ & 1 \\
\hline$\{e\}$ & 1 \\
\hline$\{f\}$ & 4 \\
\hline$\{g\}$ & 2 \\
\hline$\{h\}$ & 1 \\
\hline & \\
\hline
\end{tabular} \mid \begin{tabular}{|l|l|}
\hline Items & Support \\
\hline$\{a\}$ & 3 \\
\hline$\{b\}$ & 3 \\
\hline$\{c\}$ & 4 \\
\hline$\{f\}$ & 4 \\
\hline$\{g\}$ & 2 \\
\hline
\end{tabular}

\begin{tabular}{|c|c|c|c|}
\hline Items & $\begin{array}{l}\text { Suppor } \\
\mathrm{t}\end{array}$ & & \multirow{3}{*}{ support } \\
\hline$\{a, c, f\}$ & 3 & \multirow{2}{*}{ Items } & \\
\hline$\{a, b, c\}$ & 1 & & \\
\hline$\{a, c, b, f\}$ & 1 & $\{a, b\}$ & 1 \\
\hline$\{\mathrm{a}, \mathrm{c}, \mathrm{f}, \mathrm{g}\}$ & 1 & $\{a, c\}$ & 3 \\
\hline$\{\mathrm{a}, \mathrm{f}, \mathrm{b}\}$ & 0 & $\{a, f\}$ & 3 \\
\hline$\{\mathrm{a}, \mathrm{f}, \mathrm{g}\}$ & 1 & $\{a, g\}$ & \multirow[t]{2}{*}{1} \\
\hline$\{b, c, f\}$ & 1 & \multirow{2}{*}{$b, c$} & \\
\hline$\{b, c, f, g\}$ & 0 & & 2 \\
\hline$\{\mathrm{b}, \mathrm{f}, \mathrm{g}\}$ & 1 & $\{b, f\}$ & 2 \\
\hline \multirow[t]{4}{*}{$c, f, g$} & 1 & $\{b, g\}$ & 1 \\
\hline & & $\{c, f\}$ & 3 \\
\hline & & $\{c, g\}$ & 1 \\
\hline & & $\{f, g\}$ & 2 \\
\hline
\end{tabular}

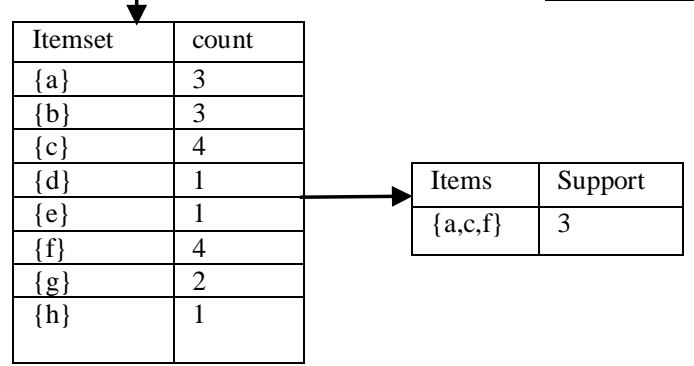

Figure 1: Steps to Derive Frequent Item Sets using Apriori

\subsection{The FP-Growth Algorithm}

The FP-tree is an extended prefix-tree structure [4] for storing compressed and crucial information about frequent patterns, while the FP-growth algorithm uses the FP-tree structure to find the complete set of frequent patterns. An FPtree consists of one root labeled as null, a set of item prefix subtrees as the children of the root and a frequent-item header table.

Each node in the prefix subtree consists of three fields:
a. Item-name
b. Count
c. Node-link

The count of a node records the number of transactions in the database that share the prefix represented by the node, and node-link links to the next node in the FP-tree carrying the same item-name. Each entry in the frequent-item header table consists of two fields:
a. Item-name
b. Head of node-link 
It points to the first node in the FPtree carrying the itemname. Besides, the FP-tree assumes that the items are sorted in decreasing order of their support counts, and only frequent items are included. After the FP-tree is built, the FP-growth algorithm recursively builds conditional pattern base and conditional for each frequent item from the FP-tree and then uses them to generate all frequent itemsets.

Table 2: Modified Transaction Database

\begin{tabular}{|c|c|c|}
\hline TID & Item bought & Ordered item \\
\hline 100 & $\mathrm{~d}, \mathrm{c}, \mathrm{a}, \mathrm{f}$ & $\mathrm{a}, \mathrm{c}, \mathrm{d}, \mathrm{f}$ \\
\hline 200 & $\mathrm{~g}, \mathrm{c}, \mathrm{a}, \mathrm{f}, \mathrm{e}$ & $\mathrm{a}, \mathrm{c}, \mathrm{e}, \mathrm{f}, \mathrm{g}$ \\
\hline 300 & $\mathrm{~b}, \mathrm{a}, \mathrm{c}, \mathrm{f}, \mathrm{h}$ & $\mathrm{a}, \mathrm{b}, \mathrm{c}, \mathrm{f}, \mathrm{h}$ \\
\hline 400 & $\mathrm{~g}, \mathrm{~b}, \mathrm{f}$ & $\mathrm{b}, \mathrm{f}, \mathrm{g}$ \\
\hline 500 & $\mathrm{c}, \mathrm{b}$ & $\mathrm{b}, \mathrm{c}$ \\
\hline
\end{tabular}

Step 1:

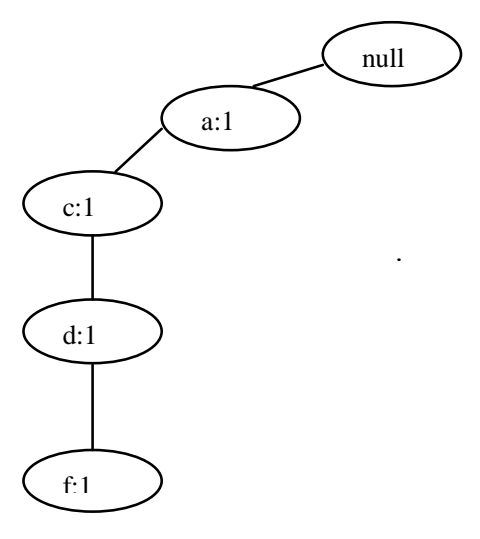

Step 2:

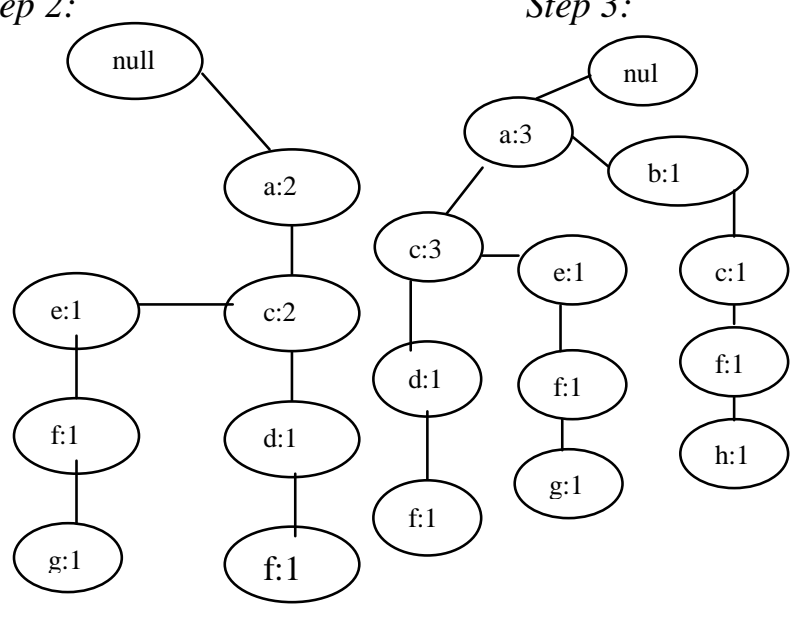

Step 4:

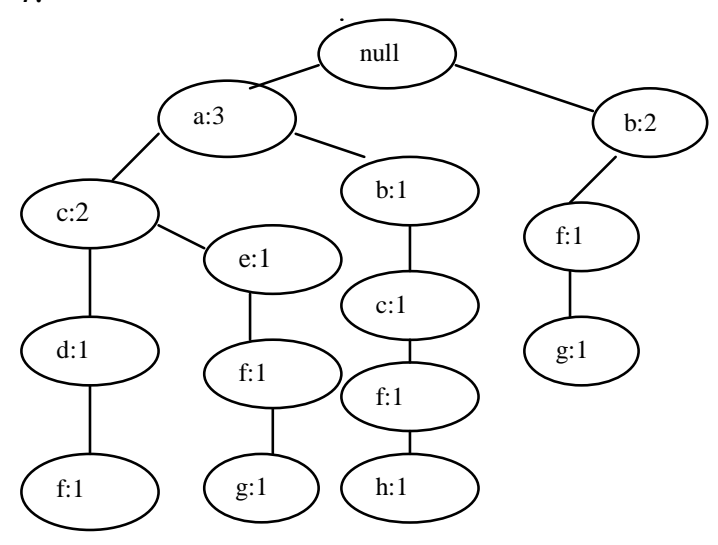

Step 5: FP-tree is created:

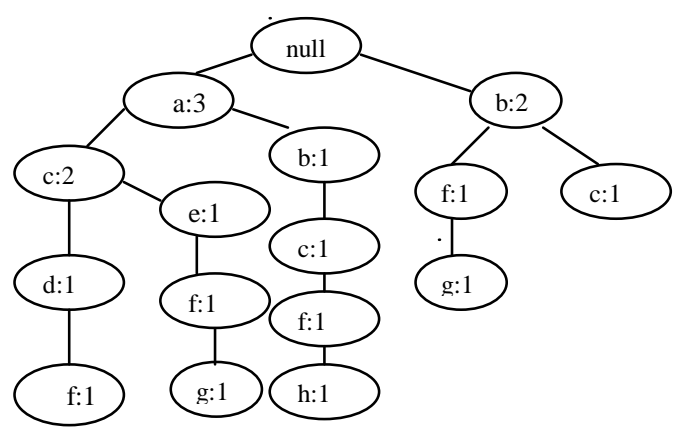

Figure 2: Construction of FP- Tree

Table 3: Generating Frequent Patterns

\begin{tabular}{|c|c|c|c|}
\hline $\begin{array}{l}\text { Ite } \\
\text { ms }\end{array}$ & $\begin{array}{l}\text { Conditional } \\
\text { pattern base }\end{array}$ & $\begin{array}{c}\text { Conditional } \\
\text { FP-tree }\end{array}$ & $\begin{array}{c}\text { Frequent } \\
\text { patterns } \\
\text { generated }\end{array}$ \\
\hline $\mathrm{a}$ & - & - & No \\
\hline $\mathrm{b}$ & - & - & No \\
\hline $\mathrm{c}$ & $\{a: 2\}\{b: 1\}\{b: 1\}$ & $\{b: 2\}$ & $\{b, c: 2\}$ \\
\hline $\mathrm{d}$ & $\{\mathrm{a}, \mathrm{c}: 1\}$ & - & No \\
\hline $\mathrm{e}$ & $\{c, a: 1\}$ & - & No \\
\hline f & $\begin{array}{c}\{\mathrm{a}, \mathrm{c}, \mathrm{d}: 1\} \\
\{\mathrm{e}, \mathrm{c}, \mathrm{a}: 1\} \\
\{\mathrm{c}, \mathrm{a}, \mathrm{a}: 1\} \\
\{\mathrm{b}: 1\}\end{array}$ & $\begin{array}{c}\{\mathrm{a}, \mathrm{c}: 3\} \\
\{\mathrm{b}: 2\}\end{array}$ & $\begin{array}{c}\{\mathrm{a}, \mathrm{c}, \mathrm{f}: 3\} \\
\{\mathrm{b}, \mathrm{f}: 2\}\end{array}$ \\
\hline $\mathrm{g}$ & $\begin{array}{c}\{\mathrm{a}, \mathrm{c}, \mathrm{e}, \mathrm{f}: 1\}\{\mathrm{f} \\
\mathrm{b}: 1\}\end{array}$ & $\{\mathrm{f}: 2\}$ & $\{f, g: 2\}$ \\
\hline $\mathrm{h}$ & $\{\mathrm{a}, \mathrm{b}, \mathrm{c}, \mathrm{f}: 1\}$ & - & No \\
\hline
\end{tabular}

\subsection{MSapriori Algorithm}

The MSapriori algorithm [12] can find rare item rules without producing a huge number of meaningless rules. In this model, the definition of the minimum support is changed. 
Each item in the database can have its minsup, which is expressed in terms of minimum item support (MIS). In other words, users can specify different MIS values for different items. By assigning different MIS values to different items, we can reflect the natures of the items and their varied frequencies in the database.

Example 1: Consider the following items in a database, bread, shoes and clothes. The user-specified MIS values are as follows:

$$
\text { MIS }(\text { bread })=2 \% ; \text { MIS }(\text { shoes })=1 \% ; \text { MIS [clothes] 0:2\% }
$$

If the support of itemset \{clothes, bread is $0.15 \%$, then itemset \{clothes, bread $\}$ is infrequent because the MIS value of itemset $\{$ clothes, bread $\}$ is equal to

min [MIS (clothes), MIS (bread)] $=0.2 \%$, which is larger than $0.15 \%$.

The task of mining association rules is usually decomposed into two steps:

(1) Frequent itemset generation: to find all frequent itemsets with supports exceeding minsup.

(2) Rule generation: to construct from the set of frequent itemsets all association rules with confidences exceeding the minimum confidence.

Note that, in order to generate the association rules from a frequent itemset, not only we need to know the support of this itemset, but the supports of all its subsets must also be known. Otherwise, it would be impossible to compute the confidences of all related rules. When there is only one single MS, the above two steps satisfy the downward closure property. That is, if an itemset is frequent, then all its subsets are also frequent. Therefore, after applying the Apriori algorithm we can find the support values of all subsets of frequent itemset $\{\mathrm{A}, \mathrm{B}, \mathrm{C}, \mathrm{D}\}$ and all related rules as well. On the contrary, when there are multiple MS, the downward closure property no longer holds. That is, some subsets of a frequent itemset may not be frequent and their supports will be missing.

\section{Algorithm MSapriori}

$1 M=\operatorname{sort}(I, M S) ; / *$ according to MIS (i)'s stored in $M S * /$

$2 F=$ init-pass $(M, T) ; / *$ make the first pass over $T * /$

$3 L 1=\{\langle f\rangle \mid f \in F, f$.count $\geq \operatorname{MIS}(f)\}$;

4 for $\left(k=2 ; L_{k}-1 \neq \Phi ; k++\right)$ do

5 if $k=2$ then $C 2=$ level2-candidate-gen $(F)$

6 else $C_{k}=$ candidate-gen $\left(L_{k}-1\right)$

7 end

8 for each transaction $t \in T$ do

$9 C_{t}=$ subset $\left(C_{k}, t\right)$;

10 for each candidate $c \in C t$ do $c$.count++;

11 end

$12 L_{k}=\left\{c \in C_{k} \mid c\right.$. count $\left.\geq M I S(\mathrm{c}[1])\right\}$

13 end

14 Answer $=\cup k L_{k}$

\section{PROPOSED SYSTEM}

\subsection{Multiple Item Support Tree (MIS-Tree)}

In this section, a new tree structure, named the MIS-tree, is proposed for mining frequent pattern with multiple MS. It is an extended version of the FP-tree structure.

A multiple item support tree is a tree structure defined as follows.

(1) It consists of one root labeled as null, a set of item prefix subtrees as the children of the root, and a MIN_frequent item header table which contains all items in F.

(2) Each node in the item prefix subtree consists of three fields: item-name, count and node link, where item-name registers which item this node presents, count registers the number of transactions represented by the portion of the path reaching this node, and node-link links to the next node in the MIS-tree carrying the same item-name, or null if there is none.

(3) Each entry in the MIN_frequent item header table consists of three fields: item-name, item's minsup MIS(ai) and head of node-link which points to the first node in the MIS-tree carrying the item-name.

(4) All the items in the table are sorted in non-increasing order in terms of their MIS values.

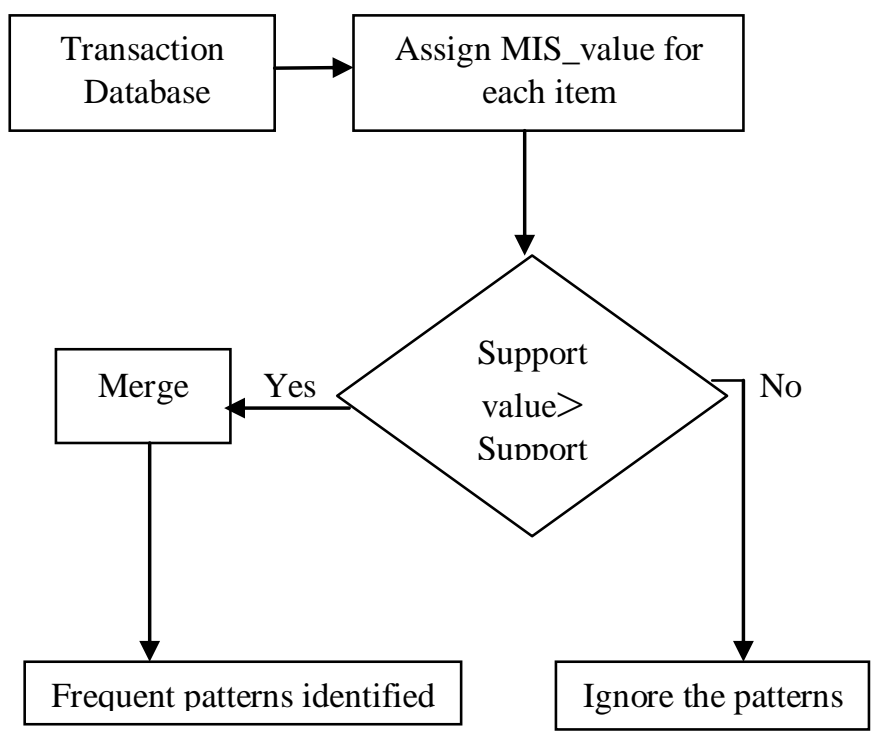

Figure 3: Flow Diagram for MIS-Tree

\section{Algorithm:}

(MIS-tree construction):

Input: A transaction database DB and a minimum support threshold of each item MIS $\left(a_{i}\right)$.

Output: Its Multiple Item Support Tree, MIS-tree.

Method:

1. Create the root of MIS-tree T, and lable it as "null" For each transaction in DB do the following:

a) Sort all items in transaction according to their MIS (ai) in nonincreasing order.

b) Count the support values of any item in transaction.

c) Let the sorted items in transaction be $(\mathrm{p}[\mathrm{p}], \mathrm{T})$, where $\mathrm{p}$ is the first element and $\mathrm{p}$ is the remaining list. Call insert tree (p[p], T). 
2. Let $\mathrm{F}$ denote the set of those items with support smaller than MIN and $f$ denote the item in F.Foe each $f$ in the following :

- Delete the entry in the header table with itemname $=f$.

- Call MIS_Pruning (Tree, $f$ ).

3. Name the resulting table as MIN frequent item header table.

4. Call MIS_Merge (Tree).

Procedure insert_tree $(\mathrm{p} / \mathrm{P}), \mathrm{T}$

While ( $\mathrm{P}$ is empty)

\{

If $\mathrm{T}$ has a child $\mathrm{N}$ such that .item-name $=\mathrm{N}$.item-name then count++;

Else

Create a new node $\mathrm{N}$, and let its count be 1 ;

Let its pattern link be linked to $\mathrm{T}$;

Let its node-link be linked to the node with same item=name via the node-link structure;

\}

Function MIS-Pruning (Tree, $\mathrm{a}_{\mathrm{i}}$ )

\{

For each node in the node-link of $a_{i}$ in Tree do \{

If the node is a leaf then remove the node directly

Else remove the node and then its parent node will be linked to its child node(s);

\}

\}

Function MIS-Merge (Tree)

\{

For each item $a_{i}$ in the MIN Frequent item header table do

\{

If there are child nodes with the same item-name then merge these nodes and set the count as the summation of these node counts;

\} \}

\section{CONCLUSION}

Efficient algorithms for mining association rules with multiple MS have presented maintenance of without rescanning database, provides an efficient algorithm for mining frequent patterns with multiple MS and it solve the problem occurred in the MSapriori algorithm that it cannot generate association rules unless a post-processing phase is executed. This method finds not only all frequent itemsets but also all subsets needed for generating association rules. Thus the results indicate that in all cases this algorithm is faster than the MSapriori algorithm.

\section{REFERENCES}

[1] R. Agrawal, R. Srikant, Fast algorithms for mining association rules, Proceedings of the 20th Very Large Databases Conference (VLDB'94), Santiago de Chile, Chile, 1994.

[2] M.S. Chen, J. Han, P.S. Yu, Data mining: an overview from a database perspective, IEEE Transactions on Knowledge and Data Engineering (1996).

[3] W. Cheung, O.R. Zaiane, Incremental mining of frequent patterns without candidate generation or support constraint, Proceedings of the 7th International Database Engineering and Applications Symposium (IDEAS'03), Hong Kong, 2003.
[4] J. Han, J. Pei, Y. Yin, Mining frequent patterns without candidate generation, Proceedings 2000 ACM-SIGMOD International Conference on Management of Data (SIGMOD’ 00), Dallas, TX, USA, 2000.

[5] H. Mannila, Database methods for data mining, Proceedings of the 4th International Conference on Knowledge Discovery and Data Mining (KDD '98) tutorial, New York, NY, USA, 1998.

[6] V. Pudi, J.R. Haritsa, Quantifying the utility of the past in mining large databases, Information Systems 25 (5) (2000).

[7] S. Thomas, S. Bodagala, K. Alsabti, S. Ranka, An efficient algorithm for the incremental update of association rules in large database, International Conference on Knowledge Discovery and Data Mining (KDD'97), Newport, CA, USA, 1997.

[8] M.C. Tseng, W.Y. Lin, Mining generalized association rules with multiple minimum supports, International Conference on Data Warehousing and Knowledge Discovery (DaWaK'01), Munich, Germany, 2001.

[9] Z. Zheng, R. Kohavi, L. Mason, Real world performance of association rule algorithms, Proceedings of the 7th ACMSIGKDD International Conference on Knowledge Discovery and Data Mining, San Francisco, CA, USA2001.

[10] R. Feldman, Y. Aumann, A. Amir, H. Mannila, Efficient algorithm for discovering frequent sets in incremental databases, Proceedings of SIGMOD Workshop on Research Issues in Data Mining and Knowledge Discovery (DMKD'97), Tucson, AZ, USA, 1997.

[11] Agarwal, R. Aggarwal, C. and Prasad V., A tree projection algorithm for generation of frequent itemsets. In J. Parallel and Distributed Computing, 2000.

[12] B. Liu, W. Hsu, Y. Ma, Mining association rules with multiple minimum supports, Proceedings of the ACM SIGKDD International Conference on Knowledge Discovery and Data Mining (KDD-99), San Diego, CA, USA, 1999. 